\title{
Improvement of Software Defined Radio based TDOA source localization
}

\author{
Junming Wei and Changbin Yu \\ Research School of Engineering \\ Australian National University \\ Canberra, Australia \\ Email: \{junming.wei, brad.yu\}@anu.edu.au
}

\begin{abstract}
In this paper, methods to improve a practical Time Difference of Arrival (TDOA) based source localization system are investigated. Firstly, our previous work on the development of a TDOA based source localization system using reconfigurable Software Defined Radios (SDRs) is described in detail. The system can achieve less than $100 \mathrm{~m}$ error in a coverage of several kilometers. However, the performance of the localization system is limited by many factors. In order to further improve the localization accuracy, a method to improve the resolution of TDOA measurements is proposed; and by investigating the synchronization error and sensor position error through experiments, their error distributions are obtained to reduce measurement error. When the measurement accuracy reaches a limit constrained by current hardware platforms, localization optimization algorithms are implemented. Instead of proving the effectiveness of the algorithms through simulation, all numerical results are obtained from real-world experiments and the results show great enhancement of the localization accuracy.
\end{abstract}

\section{INTRODUCTION}

Localization, which plays an important role in many industrial applications, is to identify location of targets in the outdoor or indoor environment. To achieve target localization, a variety of sensing techniques can be applied. The most popular outdoor localization technology is GPS. When GPS signal is not available, such as in indoor localization, other techniques can be used. For example, in mobile robot localization and navigation, especially Simultaneous Localization and Mapping (SLAM) implementation [1], [2] , 2D or 3D camera is used to obtain image information (e.g. landmarks) in the environment, and the movement of robots is usually recorded by pedometer. Laser scanners and ultrasonic sensors are also widely used to obtain range measurements in the localization [3]-[5]. Recently RF signal based localization technology also attracts many interests with the rapid development of wireless sensor network (WSN) in particular [6], [7] .

For source localization, different measurement techniques, such as Time Difference of Arrival (TDOA), Time of Arrival (TOA), Received Strength Signal (RSS) and Angle of Arrival

J. Wei was supported in part by a PhD scholarship from China Scholarship Council. This work was supported in part by the Australian Research Council through Discovery Project DP-130103610, a Queen Elizabeth II Fellowship under Grant DP110100538, and Overseas Expert Program of Shandong Province, a grant from Shandong Academy of Science Development Fund for Science and Technology, the Pilot Project for Science and Technology in Shandong Academy of Science, the Open Research Project of the State Key Laboratory of Industrial Control Technology, Zhejiang University (No.ICT 1427) and the National Natural Science Foundation of China (61375072).
(AOA) [8] can be used. Time based localization has relatively higher accuracy than other localization [9], but it is often very expensive. RSSI based localization is cheap and simple, but it has low accuracy because RSSI measurements are likely to be affected by the environment where the measurements are taken, such as temperature, humility and multi-path effect. In AOA based localization, the accuracy of AOA measurements may be influenced by ghost and hidden emitters, and measuring AOA of a signal usually requires antenna array, which is relatively complex from the point of view of system implementation.

To detect RF signal, most of platforms are developed entirely using hardware to meet a specific purpose. The disadvantages of these conventional platforms are lack of flexibility and the replacement of them (for example if the equipment has design errors, does not meet operational requirements or becomes outdated due to the availability of new technologies.) can be costly. Software Defined Radio (SDR) is a relatively new technology and becoming more and more mature. It aims to minimize the amount of specialized hardware required and implement the majority of components (and hence the desired functionality) in software. These features provide great flexibility and adaptability, and make low-cost localization solutions a reality. Therefore, it becomes a favorite platform for researches to evaluate theoretical work in practice [10]-[13].

To achieve a trade-off between the accuracy and the cost, we are particularly interested in implementing time based source localization using SDRs. Time based localization technologies include TOA based localization and TDOA based localization. The measurement of TOA needs accurate time synchronization to obtain the travel time of a radio signal from the transmitter to receivers. However, in practice the cooperation between the target and sensors is not always available. In contrast, TDOA based localization only requires time synchronization between sensors, which is more suitable for real-world localization applications than TOA based localization, especially, passive source localization. Therefore, we implemented a low-cost TDOA based source localization system using SDRs which can localize with less than $100 \mathrm{~m}$ localization error in a coverage of several kilometers.

The performance of a localization system can be improved from at least two aspects. Firstly, some methods [14], [15] can be used to reduce measurement errors. Secondly, since noise in the measurements is inevitable, localization optimization algorithms [16]-[18] can also be implemented. In this paper, we implement both measurement error reduction and localization 
optimization solutions to further enhance the accuracy of the SDR based TDOA localization system.

The contributions of this paper are twofold: 1) we detail the design and implementation of a TDOA based source localization system developed using Software Defined Radios. The difference between our work and [19], [20] is that we developed a large-scale localization system with a range of several kilometers rather than a small area. Moreover, the signal source we localize is completely passive (i.e. not under our control), which shows the localization system has good scalability to localize a wide range of signal source. 2) We systematically analyze and address different factors that affect the performance of the localization system, ranging from the resolution of TDOA measurements, synchronization error and sensor position error. In addition, when the measurement accuracy reaches a limit constrained by the hardware platform, localization optimization methods are implemented and their performances are evaluated through experiments.

The rest of the paper is organized as follows: The TDOA based localization and the implementation of the low-cost TDOA based source localization system developed using Software Defined Radios are revisited in Section II and Section III. Section IV analyses the constraints that limit the measurement accuracy of this localization system and the corresponding solutions are proposed. Section V compares localization optimization algorithms and obtains numerical results using realworld data. In Section VI, the conclusion and future work are given.

\section{TDOA BASED LOCALIZATION}

We start with briefly reviewing the principle of TDOA based localization [21]. TDOA based localization system, also known as hyperbolic localization system, is to determine the position of an emitter from measurements of the time differences of arrival of the emitter's signal at pairs of receivers whose location are known. In the noiseless case the localization problem can be formulated as follows. Suppose there is an emitter or a target whose coordinate vector is $\boldsymbol{x}=\left(x_{1}, x_{2}, \ldots, x_{n}\right)^{T}$. Further, $\boldsymbol{\Theta}=\left(\theta_{1}, \theta_{2}, \ldots, \theta_{N}\right)^{T}$ denotes a set of measurements obtained from sensors. So we have

$$
\Theta=\boldsymbol{f}(\boldsymbol{x})
$$

where $\boldsymbol{f}=\left(f_{1}, f_{2}, \ldots, f_{N}\right)^{T}$ denotes the mapping from the target position to the measurements. The function $f$ can be obtained analytically according to the geometry of the target and sensors.

In TDOA based localization in 2-dimensional space $(n=$ 2 ), at least three physical sensors are required to localize a single target. We can obtain only two TDOA measurements $(N=2)$ from the synchronized sensors according to the arrangement of Fig. 1 as follows.

$$
\begin{aligned}
& t_{2}-t_{1}=f_{1}(x, y)=\frac{d_{2}-d_{1}}{c} \\
& t_{3}-t_{1}=f_{2}(x, y)=\frac{d_{3}-d_{1}}{c}
\end{aligned}
$$

where $t_{i}$ denotes the time when the signal from the emitter arrives at the sensor $i . c$ is a known constant speed of signal propagation. $d_{i}$ denotes Euclidean distance between the emitter and the sensor $i\left(d_{i}=\sqrt{\left(x-x_{i}\right)^{2}+\left(y-y_{i}\right)^{2}}\right)$.

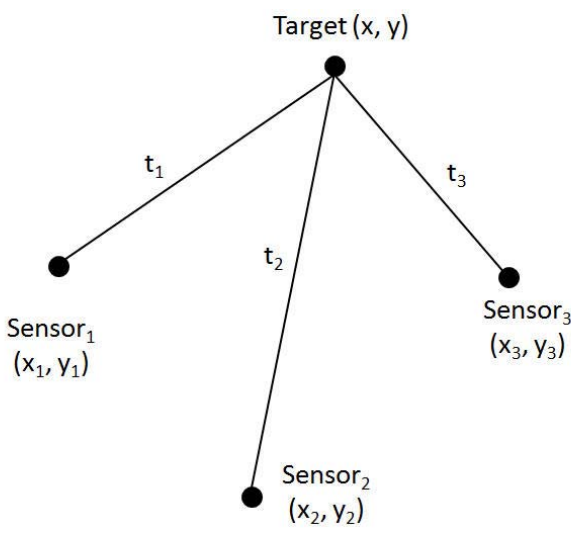

Fig. 1. TDOA based localization with two measurements in 2D

However, in practice, noise in the measurement is inevitable. Therefore, the mapping from the target position to the measurements can be described by a nonlinear equation as follows (we use $\tilde{\boldsymbol{\Theta}}=\left(\tilde{\theta}_{1}, \tilde{\theta}_{2}, \ldots, \tilde{\theta}_{N}\right)$ to denote the noisy measurements):

$$
\tilde{\boldsymbol{\Theta}}=\boldsymbol{f}(\boldsymbol{x})+\delta \boldsymbol{\Theta}
$$

where $\delta \boldsymbol{\Theta}=\left(\delta \theta_{1}, \delta \theta_{2}, \ldots \delta \theta_{N}\right)^{T}$ denotes the noise in the measurements.

When $N=n$ one obtains a target position estimate in effect by solving $\Theta=\boldsymbol{f}(\boldsymbol{x})$. However, generally when $N \geq$ $n+1$, this equation will have no solution in the noisy case. In order to obtain an approximate position estimate, various methods have been proposed [22], [23]. The main idea of these approaches is similar: convert the localization problem to an optimization problems as follows.

$$
\tilde{\boldsymbol{x}}=\arg \min _{\boldsymbol{x}} F_{\text {cost-function }}(\boldsymbol{x}, \tilde{\boldsymbol{\Theta}})
$$

where $\tilde{\boldsymbol{x}}=\left(\tilde{x}_{1}, \tilde{x}_{2}, \ldots, \tilde{x}_{n}\right)^{T}$ denotes the inaccurate target position estimate. By solving the above equation, which is often computationally difficult, we obtain the estimated position.

\section{THE IMPLEMENTATION OF TDOA BASED LOCALIZATION SYSTEM USING SDRS}

Firstly, the TDOA based localization system developed using SDRs [24], [25] is revisited. The SDR model we used is USRP N210 which is developed by Ettus Research [26]. Each USRP N210 consists of RF front ends (daughterboards) to capture signal, a FPGA and ADC/DACs for simple signal processing and sampling. It needs to work with a host computer and antennas. The signal processing that would traditionally have been carried out by dedicated hardware (e.g. mixers, tuners, amplifiers, filters, etc.) is handled by the PC's general purpose processor. Moreover, the characteristics of RF sensor developed on SDRs, such as central frequency, bandwidth, modulation scheme, coding rate, etc., can even be changed during runtime according to software configuration. Opensource software, GNU Radio [27], is used for programming. It includes many available blocks that handle a wide range of signal processing functions and enables developers to add extra modules for specific applications.

To demonstrate the TDOA based localization in practice, we developed a TDOA based localization system using SDRs, 


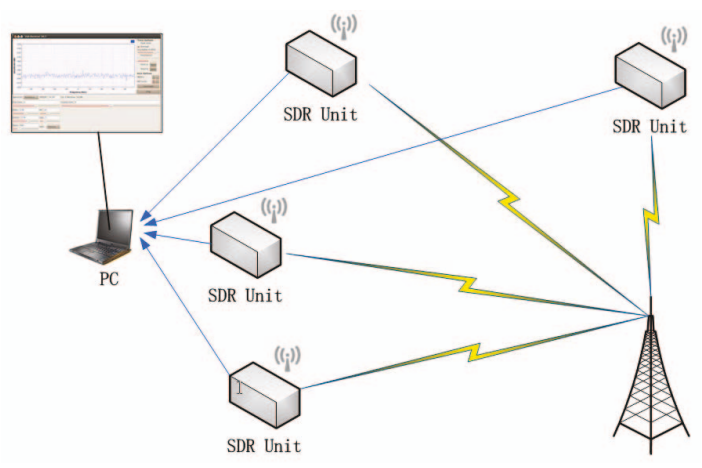

Fig. 2. The framework of the TDOA based localization system using SDRs [24]

as shown in Fig. 2. There are two key problems need to be tackled in order to localize an emitter using SDRs. One is how to measure time difference of signal arrival using SDR pairs. The other one is how to achieve accurate synchronization between multiple SDR receivers.

\section{A. Obtain TDOA measurements}

The signal transmitted from a remote source at two spatially separated sensors can be mathematically modeled as [28]

$$
\begin{array}{r}
r_{1}(n)=s(n)+w_{1}(n) \\
r_{2}(n)=s(n-D)+w_{2}(n) \\
n=0,1, \ldots, N-1
\end{array}
$$

where $s(n), w_{1}(n)$ and $w_{2}(n)$ are independent zero-mean white Gaussian variables, $N$ is the length of sampling. Digitalized signal $s(n)$ is assumed to be uncorrelated with noise $w_{1}(n)$ and $w_{2}(n) . D$ is the delay of sampling points to be estimated, which is related to the TDOA measurements obtained by two SDR receivers. $\hat{D}$ can be obtained by maximizing the cross-correlation between $r_{1}(n)$ and $r_{2}(n)$, that is

$$
\begin{array}{r}
\hat{D}=\arg \max _{\tilde{D}} J(\tilde{D}) \\
J(\tilde{D})=\sum_{n=0}^{N-1} r_{1}(n-\tilde{D}) r_{2}(n)
\end{array}
$$

In the SDR based TDOA localization system, the digitalized signal received by a pair of SDRs is processed through cross correlation and the TDOA is equal to $\frac{1}{f_{s}} * \hat{D}\left(f_{s}\right.$ is the sampling rate of the SDR sensors).

\section{B. Synchronization between multiple SDRs}

Accurate synchronization between receivers is critical to obtain accurate TDOA measurements. In general, the common timescale for local clocks of sensors in a network can be achieved in two methods, distributed and centralized methods. Distributed synchronization is usually obtained through message exchanges between sensors. However, the messages carrying time stamps always suffer from various delays, such as send time delay, access time delay, propagation time delay and receive time delay [29]. In addition, synchronization through exchanging messages may be well applied to shortrange localization, but when sensors are spatially distributed in a large area, a centralized method is suitable.

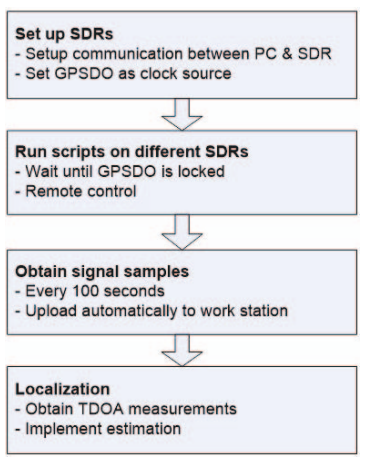

Fig. 3. Work procedure of the localization system

In this application, the synchronization of SDRs is achieved through GPSDO modules. The roles of the GPSDO are two aspects: firstly, it disciplines the frequency output of the SDR sensor. The oscillator of the USRP is TCXO-based and it provides $2.5 \mathrm{ppm}$ frequency accuracy. After the GPSDO module is used, the frequency accuracy of the USRP is enhanced to $0.01 \mathrm{ppm}$ which is equivalent to $+/-0.1 \mathrm{~Hz}$ frequency error. Therefore, GPSDO improves the frequency accuracy of the SDR significantly. Secondly, GPSDOs provide time reference to spatially distributed SDR sensors. The 1PPS time reference outputs from GPSDOs is synchronized to Coordinated Universal Time (UTC) and the achieved 1PPS accuracy is tens of nanoseconds, which gives error up to $30 \mathrm{~m}$ in distance. While time issued by GPS receivers has some error, they are widely used to synchronize spatially distributed sensors outdoors because this is a low-cost, reliable and relatively accurate solution.

During the target localization, after SDRs are synchronized through GPSDOs, we start to sample the signal at the same time using a pair of SDRs. Then, the cross correlation is implemented on received samples to obtain TDOA measurements. The process of localization is implemented in a centralized way and the work procedure of the localization system is shown in Fig 3.

1) Deploy SDRs at different locations and set up the communication between host PCs and SDRs.

2) After the synchronization is achieved between different SDRs through GPSDOs, the program on each SDR can be executed through remote control. The programs can detect time setting so that multiple SDRs start to sample at the same time.

3) Signal sampling is implemented by multiple SDRs every 100 seconds and the stored signals at each SDR are uploaded to a work station automatically.

4) At the work station, the TDOA measurements are obtained through cross correlation. Then, the target location is estimated.

\section{REDUCTION OF SDR MEASUREMENTS ERROR AND SENSOR POSITION ERROR}

In our previous work [24], the factors that influence the performance of the SDR based TDOA localization system were summarised without detailed analysis. Here, we focus on studying solutions to reduce SDR measurements and sensor position error. Firstly, methods to enhance the resolution of 
TABLE I. COMPARISON OF TIME DELAY ESTIMATION METHODS

\begin{tabular}{|l|l|}
\hline Methods & Results (samples) \\
\hline Cross correlation & e.g. 153 \\
\hline Parabolic fit interpolation & 152.4999940881971 \\
\hline Zero crossing of Hilbert transformation & 153.4475526848109 \\
\hline Hilbert Transform of FFT Pruned Correlation & 153.1673833503155 \\
\hline
\end{tabular}

TDOA measurements are evaluated. Secondly, since this is a time based (TDOA) localization system, the accuracy of time synchronization of multiple SDRs is critical to obtain accurate TDOA measurements. In addition, sensor position error will cause target location estimation error. Therefore, we obtain the distribution of synchronization error and sensor position error through experiments to improve the localization performance.

\section{A. Improvement of TDOA resolution}

TDOA measurements are obtained through the cross correlation of sampled signals, so the resolution of TDOA measurements is limited by the sampling rate (frequency) of the SDR. For example, if the highest sample rate $f_{s}=25 \mathrm{M}$ sps achieved by USRP N210s is used, the time interval between two successive digitalized samples is $T_{s}=1 / f_{s}=40 \mathrm{~ns}$, which corresponds to $12 \mathrm{~m}$ resolution in distance. To enhance the resolution of TDOA measurements, optimized time delay estimation algorithms are required to achieve sub-sample level accuracy in cross correlation. Table I shows the performance of different time delay estimation algorithms using experimental data.

One of widely used methods to achieve optimized time delay estimation is parabolic fit interpolation which locates optimized peak of the cross correlation function, but it has high bias [30]. Here, we employ the zero crossing of Hilbert transformation of FFT pruned correlation [31] to enhance the resolution of TDOA measurements. This method takes the advantage of both FFT pruning and Hilbert transform. The FFT pruning is implemented by appending zero between the new and old Nyquist frequency in transform domain, which is equivalent to increasing the sampling frequency in the time domain after the inverse Fourier transform. The Hilbert transform can improve the TDOA measurements because when the cross correlation passes through a maximum, its Hilbert transform will cross zero, as shown in Fig. 4. In real-world measurement, the received signal usually does not hit the multiple of sampling instant during a sampling process by ADC. As a result, the zero crossing may occur at a point not explicitly defined by the data points. Therefore a simple linear interpolation between two data points which are the local minimum and maximum lie below and above zero respectively can be employed in order to locate the actual location for the delay estimates. Another advantage of using this technique is when the peak is fairly broad, especially for narrow-band signal, we do not have to take the derivative of the cross correlation function to locate the maximum. This enables a very simple search process to be used to determine the time delay. After the Hilbert transform of FFT pruning algorithm is implemented on signals obtained by a synchronized SDR pair, the resolution of TDOA measurement is enhanced from $6120 \mathrm{~ns}$ to $6125.2258 \mathrm{~ns}$, for example, which gives higher time and distance resolution.
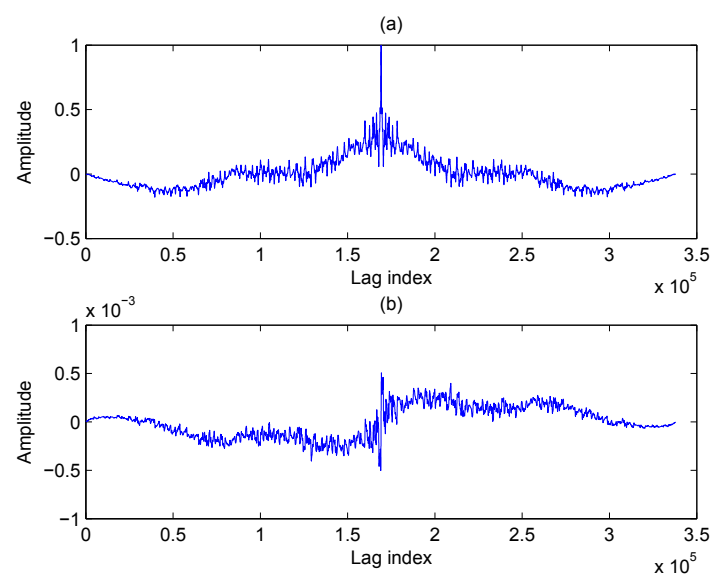

Fig. 4. (a) Cross correlation (b) Hilbert Transformed Cross correlation

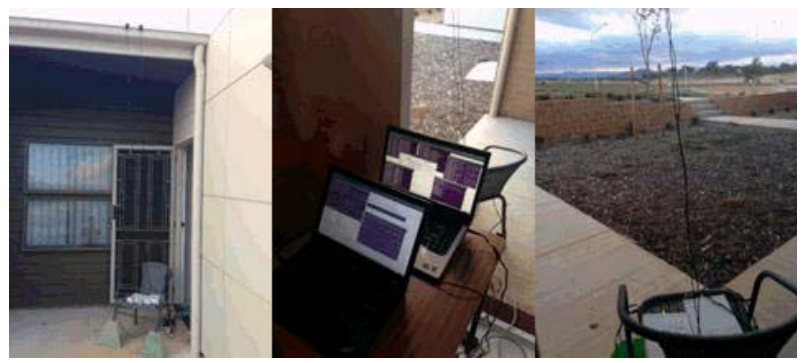

Fig. 5. Experimental setting up for the investigation of synchronization error

\section{B. Investigation of synchronization error}

Time synchronization error between sensors will produce TDOA measurement error and thus influence the localization accuracy. To investigate the synchronization accuracy in the experiments, two co-located SDR receivers were used in order to minimize the influence of other errors, such as multi-path effect, thus focusing on synchronization error only. The setting up of the experiments is shown in Fig. 5. The GPS antennas are attached to the roof to make sure they have a good view to the sky. Two host PCs control two SDRs. The influence of non-line-of-sight is also reduced because both of the receiver antennas face to the emitter which locates on the top of the mountain several kilometers away. TDOA measurements are obtained every 100s. The result obtained by more than 100 TDOA measurements using two SDR sensors shows that the synchronization error, measured in samples, obeys Gaussian distribution approximately in Fig. 6. The mean synchronization error is 1.1171875 samples, which corresponds to $44.6875 \mathrm{~ns}$ and $13.41 \mathrm{~m}$ with the $25 \mathrm{M}$ sps sampling rate. The standard deviation of the synchronization error is 5.7296 samples. This synchronization error is the combined effect of frequency and time error of the GPSDO modules. In the real-world localization implementation, the synchronization error between spatially separated SDR sensors may be slightly larger because of multi-path effect. To obtain high synchronization accuracy, high-quality GPSDO can be used but the cost will increase as well. A trade-off can be made by implementing optimized algorithms to estimate the synchronization error and correct TDOA measurements. 


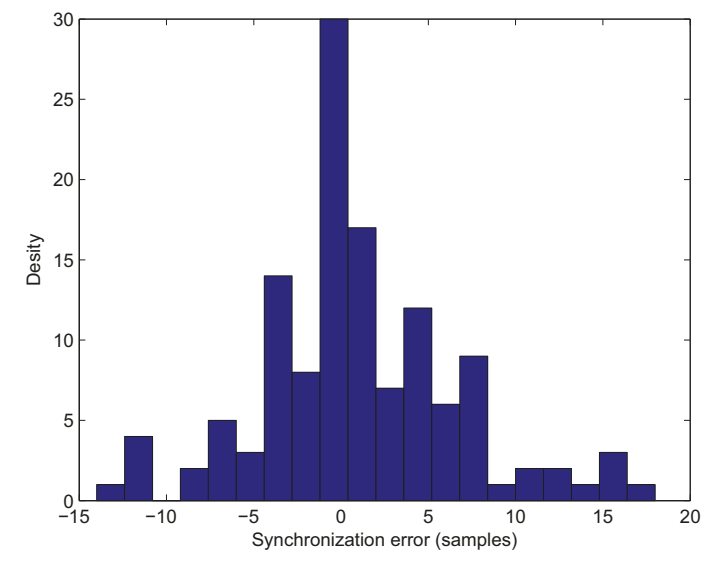

Fig. 6. The statistic of synchronization error
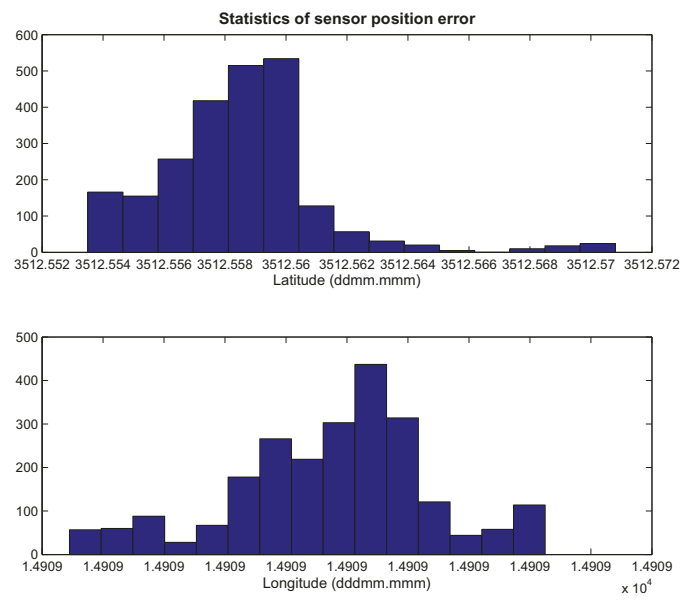

Fig. 7. Statistics of sensor location error

\section{Investigation of sensor position error}

Sensor position error will cause target location estimation error. The GPSDOs are able to give the position of stationary SDR sensors after they are deployed at different positions. However, it is well known that the position output of commercial GPS receivers is not accurate due to many error sources, such as the temporary interference of buildings or trees with line-of-sight transmission from satellites and poor satellite geometry, etc. To investigate the position accuracy of the GPSDO module, all the outputs of GPSDO NMEA sentences are recorded to obtain the statistics of GPSDO position error. The measurement took about 6 hours and 2400 measurements were obtained. As shown in Fig. 7, the variance of sensor position error is small, 7.411957e-006' in latitude and $9.978486 \mathrm{e}-006$ ' longitude, which are equal to $4.66 \mathrm{~m}$ and $5.08 \mathrm{~m}$ in distance. In real-world experiments, to obtain SDR sensor position as accurate as possible, the position that have minimum mean square error (MMSE) among multiple measurements is used as the sensor position.

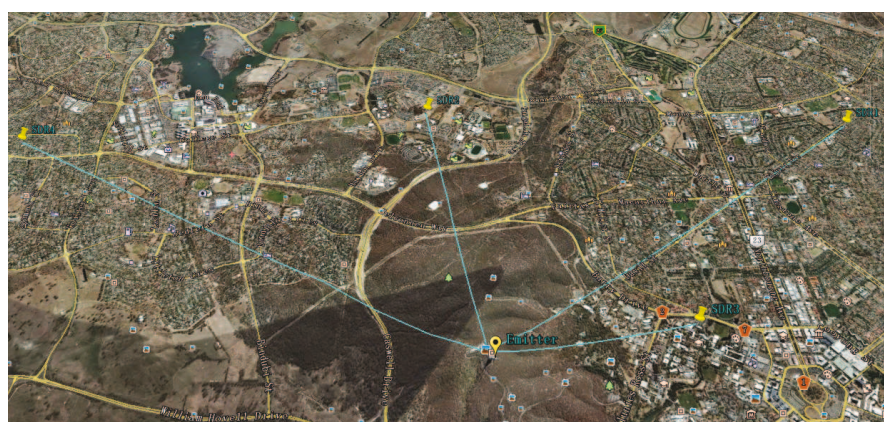

Fig. 8. Deployment of the emitter and 4 receivers

\section{IMPROVEMENT OF LOCATION ESTIMATE IN THE PRESENCE OF NOISY MEASUREMENTS}

While measurement and sensor position error can be reduced, they cannot be removed in real-world applications. To enhance the accuracy of the localization system, apart from using error reduction methods, how to process the obtained measurements using optimized localization algorithms is also important.

To practically implement passive source localization, a suitable emitter needs to be found. While a SDR sensor can be programmed to be a transmitter, its power is not high enough for long-distance transmission. A self-made powerful emitter may violate the local RF signal rules. Therefore, a local FM radio station was chosen to verify the performance of the SDR based TDOA localization system because FM radio is free and powerful enough for long-distance experiments.

Four SDR receivers were deployed at different locations with GPS coordinates obtained from GPSDOs, SDR1 ($\left.35.2531^{\circ}, 149.1503^{\circ}\right), \operatorname{SDR} 2\left(-35.2435^{\circ}, 149.0975^{\circ}\right)$, SDR3 $\left(-35.2741^{\circ}, 149.1203^{\circ}\right), \operatorname{SDR} 4\left(-35.2408^{\circ}, 149.0463^{\circ}\right)$, as shown in Fig. 8. Here we only consider 2D space for simplicity. The distances between the emitter and receivers are about $5.4 \mathrm{~km}, 3.6 \mathrm{~km}$ and $2.0 \mathrm{~km}, 6.1 \mathrm{~km}$ respectively. Take SDR1 as the reference, denote $t 21, t 31$ and $t 41$ as the three TDOA measurements obtained by SDR2 and SDR1, SDR 3 and SDR1, SDR4 and SDR1. In 2D space, after $t 21, t 31$ and $t 41$ are obtained, the localization of the emitter can be estimated. In the following parts, the methods to achieve optimized location estimation are discussed.

\section{A. Closed-form least-squares solution}

To obtain localization solution using TDOA measurements, while Maximum Likelihood Estimator (MLE) can achieve CRLB performance, it needs a good initial estimate of the target location which is usually the true target position. In real-world localization applications, however, the true target position is not available. Therefore, the least-squares based estimator is more suitable for practical applications as it can provide closed-formed solution to TDOA based localization. In this localization system, the two-phase least-squares (2WLS) solution [32] is implemented. The performance of this estimator is shown in Fig. 9. By using SDR positions, TDOA measurements and covariance matrix of TDOA measurements, the emitter location can be obtained, which gives about $53.1194 \mathrm{~m}$ localization error. 


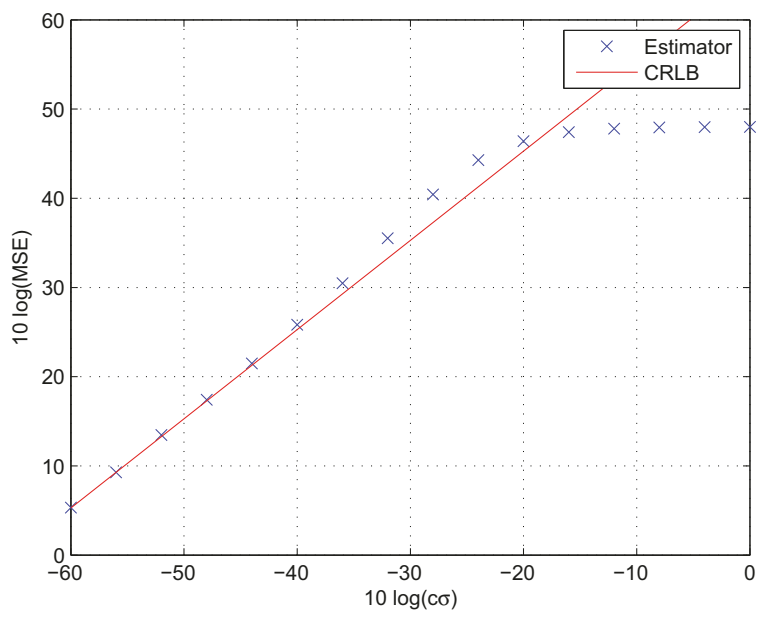

Fig. 9. Performance of the closed-formed LS estimator

A quick investigation on the noise power may give some explanation of the localization estimation error. By comparing the mean measurement errors and true TDOA values, the noise power of TDOA measurements obtained from pairs of SDRs can be calculated. The averaged noise power of $t 21, t 31$ and $t 41$ are $-15.1914 \mathrm{~dB},-19.4362 \mathrm{~dB}$ and $-10.8058 \mathrm{~dB}$ respectively, which falls in the noise range of $-20 \sim-10 \mathrm{~dB}$ of the estimator. Therefore, the implemented solution can provide a closedformed solution to this localization system, but it is not the best estimator because the noise power of the TDOA measurements falls in the region where the estimator cannot achieve CRLB performance.

\section{B. Closed-form location estimation in the presence of sensor position error}

As we discussed before, the localization error is mainly caused by synchronization error and sensor position error. Here, an algorithm which considers the existence of sensor position error [33] is also implemented. The localization error is reduced from $53.1194 \mathrm{~m}$ to $52.1 \mathrm{~m}$. Although this algorithm produces almost similar localization accuracy, it shows a slightly better performance. The main reason why this algorithm cannot give significate enhancement of localization accuracy is that the noise power of the sensor position error is very small. According to our investigation, the distance standard deviations of the sensor position obtained from GPSDOs are about $4.66 \mathrm{~m}$ in latitude and $5.08 \mathrm{~m}$ in longitude. Based on the range from the SDR receivers to the emitter in our implementation, the distance noise power produced by sensor position error is about $-24 \mathrm{~dB} \sim-30 \mathrm{~dB}$. Therefore, only correcting the sensor position error in the target location estimation does not contribute a lot to the enhancement of localization accuracy.

\section{Location estimation by correcting synchronization error}

Synchronization error is a dominate factor that influences the accuracy of TDOA measurements and thus the localization results. By effectively estimating synchronization error, the TDOA measurements can be corrected and so the accuracy of the localization system can be enhanced. Here, a statistic
TABLE II. COMPARISON BETWEEN SDR BASED LOCALIZATION AND OTHER COMMERCIAL LOCALIZATION SYSTEMS

\begin{tabular}{|l|l|l|l|}
\hline System & Coverage & Accuracy or Error $\%$ & Cost \\
\hline Locata [34] & $5 \mathrm{~km} * 5 \mathrm{~km}$ (standard config.) & Centimeter level & High \\
\hline Ubisense [35] & $20 \mathrm{~m} * 20 \mathrm{~m}(4$ sensors) & $1.5 \%$ & High \\
\hline PinPoint [36] & $150 \mathrm{~m} * 150 \mathrm{~m}$ & $0.5 \%$ & Low \\
\hline SDR & $5 \mathrm{~km} * 5 \mathrm{~km}$ & $1 \%$ & Low \\
\hline
\end{tabular}

method is used to correct TDOA measurements. Based on the distribution of synchronization error obtained in real-world experiments in Section III, the mean value of synchronization error is used to correct all TDOA measurements. While in practice, the distribution of synchronization error obtained by different pairs of SDRs may be different, the difference is small as the GPSDO modules used on each SDR sensor are the same. Therefore, we assume the mean of synchronization error is the same as the previous value obtained in experiments for all pairs of SDRs. After the TDOA measurements are corrected, the localization error is reduced to $32.8619 \mathrm{~m}$.

\section{Comparison with other time based localization systems}

A comprehensive comparison of the TDOA based localization system developed using Software Defined Radios and other time based commercial localization systems considering the coverage, accuracy and cost is shown in Table II. Locate is a GPS-like localization system. A node receives signal from multiple transmitters which are accurately synchronized through TimeLoc methodology and achieves self-localization using TOA measurements. The performance of Locata is outstanding with large coverage and very high localization accuracy, but it is very expensive. Ubisense uses different principle from Locata. Ubitags (transmitters) are localized by a network of Ubisensors that are fixed in known positions through a combination of TDOA and AOA measurements. The synchronization of Ubisensors is achieved by cable connection which limits the coverage of the system. It can achieve $1.5 \%$ error percentage. Nodes in PinPoint are localized using range measurements obtained from TOA measurements. The coverage of this system is small because the TOAs are obtained through time-stamp message exchange between two nodes. It is a low-cost localization system which gives $0.5 \%$ error percentage. The localization system developed in this paper is also a low-cost localization solution. It has large coverage and can achieve $1 \%$ error percentage. Therefore, the SDR based TDOA localization system has comparable localization performance to some commercial localization systems.

\section{CONCLUSION}

In this paper, we present the design and implementation of a TDOA based localization system developed using SDRs in detail. To improve the performance of the localization system, both measurement error reduction and localization optimization algorithms are implemented. Firstly, optimized time delay estimate algorithm is implemented to enhance the resolution of TDOA measurements. The distributions of synchronization error and sensor position error of the localization system are also obtained through experiments to reduce measurements error. In TDOA based target location estimation, localization optimization algorithms are implemented. By considering sensor position error and synchronization error in the location 
estimation, the localization accuracy is further enhanced. Numerical results obtained from experimental data show that the combination of the proposed methods can effectively correct measurement error and improve the performance of the localization system greatly.

Our future work will focus on further enhancing the accuracy of the localization system. Firstly, since the RF parameters of SDRs can be simply changed through software, optimized parameters will be obtained to reduce measurement error. Secondly, apart from taking synchronization error and sensor position error into consideration during the target location estimation, other factors such as geometric distribution of sensors, localization bias, etc. will also be studied.

\section{REFERENCES}

[1] B. Vincke, A. Elouardi, A. Lambert, and A. Merigot, "Efficient implementation of EKF-SLAM on a multi-core embedded system," in 38th Annual Conference on IEEE Industrial Electronics Society (IECON), 2012, pp. 3049-3054.

[2] G. Tuna, K. Gulez, V. Cagri Gungor, and T. Veli Mumcu, "Evaluations of different simultaneous localization and mapping (SLAM) algorithms," in 38th Annual Conference of IEEE Industrial Electronics Society (IECON), 2012, pp. 2693-2698.

[3] J. Velagic, A. Kaknjo, M. Hujdur, F. Dautovic, and N. Osmic, "Localization of holonomous mobile robot HOLBOS using extended Kalman filter (EKF) and robotic vision," in 39th Annual Conference of the IEEE Industrial Electronics Society (IECON), 2013, pp. 4180-4185.

[4] M. Cao and H. Hashimoto, "Specific person recognition and tracking of mobile robot with Kinect 3D sensor," in 39th Annual Conference of the IEEE Industrial Electronics Society (IECON), 2013, pp. 8323-8328.

[5] V.-D. Hoang, M.-H. Le, D. C. Hernandez, and K.-H. Jo, "Localization estimation based on Extended Kalman filter using multiple sensors," in 39th Annual Conference of the IEEE Industrial Electronics Society (IECON), 2013, pp. 5498-5503.

[6] A. Azenha, L. Peneda, and A. Carvalho, "A neural network approach for radio frequency based indoors localization," in 38th Annual Conference of IEEE Industrial Electronics Society (IECON), 2012, pp. 5990-5995.

[7] H. Song, V. Shin, and M. Jeon, "Mobile node localization using fusion prediction-based interacting multiple model in cricket sensor network," IEEE Transactions on Industrial Electronics, vol. 59, no. 11, pp. 43494359, 2012.

[8] N. Patwari, J. N. Ash, S. Kyperountas, A. O. Hero, R. L. Moses, and N. S. Correal, "Locating the nodes: cooperative localization in wireless sensor networks," IEEE Signal Processing Magazine, vol. 22, no. 4, pp. $54-69,2005$.

[9] R. Zekavat and R. M. Buehrer, Handbook of position location: Theory, practice and advances. NJ Wiley, 2012, vol. 27.

[10] J. Zhang, J. Jia, Q. Zhang, and E. M. K. Lo, "Implementation and evaluation of cooperative communication schemes in Software-Defined Radio testbed," in Proceedings of IEEE 2010 INFOCOM, 2010, pp. 5618-5621.

[11] M. Rahman, H. Baidoo-Williams, R. Mudumbai, and S. Dasgupta, "Fully wireless implementation of distributed beamforming on a Software-Defined Radio platform," in ACM International conference on Information Processing in Sensor Networks, 2012, pp. 305-316.

[12] H.-C. Chen, T.-H. Lin, H. Kung, C.-K. Lin, and Y. Gwon, "Determining RF angle of arrival using COTS antenna arrays: a field evaluation," in Milltary Communications Conference (MILCOM), 2012, pp. 1-6.

[13] J. Wei, Y. Ji, and C. Yu, "Improvement of Software Defined Radio based RSSI localization with bias reduction," in Proceedings of the 19th World Congress, 2014.

[14] H.-S. Ahn and K. H. Ko, "Simple pedestrian localization algorithms based on distributed wireless sensor networks," IEEE Transactions on Industrial Electronics, vol. 56, no. 10, pp. 4296-4302, 2009.

[15] H. Cho and S. W. Kim, "Mobile robot localization using biased chirpspread-spectrum ranging," IEEE Transactions on Industrial Electronics, vol. 57, no. 8, pp. 2826-2835, 2010.
[16] H. Guo, K.-S. Low, and H.-A. Nguyen, "Optimizing the localization of a wireless sensor network in real time based on a low-cost microcontroller," IEEE Transactions on Industrial Electronics, vol. 58, no. 3, pp. 741-749, 2011.

[17] B. L. Daku and J. E. Salt, "Directional performance of an algorithm used to locate microseismic events in underground mines," in 37th Annual Conference on IEEE Industrial Electronics Society (IECON), 2011, pp. 2198-2201.

[18] W. Li, J. Portilla, F. Moreno, G. Liang, and T. Riesgo, "Improving target localization accuracy of wireless visual sensor networks," in 37th Annual Conference on IEEE Industrial Electronics Society (IECON), 2011, pp. 3814-3819.

[19] N. El Gemayel, S. Koslowski, F. K. Jondral, and J. Tschan, "A low cost TDOA localization system: Setup, challenges and results," in 10th Workshop on Positioning Navigation and Communication (WPNC), 2013, pp. 1-4.

[20] J. Bhatti, T. Humphreys, and B. Ledvina, "Development and demonstration of a TDOA-based GNSS interference signal localization system," in IEEE/ION Position Location and Navigation Symposium (PLANS), 2012, pp. 455-469.

[21] Y. Ji, C. Yu, and B. Anderson, "Systematic bias correction in source localization," IEEE Transactions on Aerospace and Electronic Systems, vol. 49, no. 3, pp. 1692-1709, 2013.

[22] W. Foy, "Position-location solutions by taylor-series estimation," IEEE Transactions on Aerospace and Electronic Systems, no. 2, pp. 187-194, 1976.

[23] D. Torrieri, "Statistical theory of passive location systems," IEEE Transactions on Aerospace and Electronic Systems, no. 2, pp. 183-198, 1984.

[24] J. Wei and C. Yu, "Performance analysis of TDOA based localization using SDRs," in 3rd Australian Control Conference (AUCC), 2013, pp. 91-92.

[25] J. Wei, Y. Ji, and C. Yu, "Experimental verification of bias reduction in TDOA based localization," in 11th international conference on Control and Automation (ICCA), 2013.

[26] "Ettus Research," [Online]. Availabe: http://www.ettus.com/.

[27] "GNU Radio Wiki," [Online]. Availabe: http://gnuradio.org/redmine/projects/gnuradio/wiki.

[28] H. So, Y. Chan, K. Ho, and Y. Chen, "Simple formulae for bias and mean square error computation," IEEE Signal Processing Magazine, vol. 30 , no. 4 , pp. $162-165,2013$.

[29] F. Sivrikaya and B. Yener, "Time synchronization in sensor networks: a survey," IEEE Network, vol. 18, no. 4, pp. 45-50, 2004.

[30] C. Knapp and G. Carter, "The generalized correlation method for estimation of time delay," IEEE Transactions on Acoustics, Speech and Signal Processing, vol. 24, no. 4, pp. 320-327, 1976.

[31] N. S. M. Tamim and F. Ghani, "Techniques for optimization in time delay estimation from cross correlation function," International Journal of Engineering and Technology, vol. 10, pp. 69-75, 2010.

[32] Y. Chan and K. Ho, "A simple and efficient estimator for hyperbolic location," IEEE Transactions on Signal Processing, vol. 42, no. 8, pp. 1905-1915, 1994.

[33] K. Ho, X. Lu, and L.-o. Kovavisaruch, "Source localization using TDOA and FDOA measurements in the presence of receiver location errors: analysis and solution," IEEE Transactions on Signal Processing, vol. 55, no. 2, pp. 684-696, 2007.

[34] J. Barnes, C. Rizos, J. Wang, D. Small, G. Voigt, and N. Gambale, "Locata: a new positioning technology for high precision indoor and outdoor positioning," in International Symposium on GPS $\backslash$ GNSS, 2003, pp. $9-18$.

[35] P. Steggles and S. Gschwind, "The ubisense smart space platform," in Adjunct Proceedings of 2005 International Conference on Pervasive Computing, vol. 191, 2005, pp. 73-76.

[36] M. Youssef, A. Youssef, C. Rieger, U. Shankar, and A. Agrawala, "Pinpoint: An asynchronous time-based location determination system," in 4th international conference on Mobile systems, applications and services, 2006, pp. 165-176. 